\title{
Smart bandages: The future of wound care
}

\author{
Hossein Derakhshandeh ${ }^{1}$, Sara Saheb Kashaf ${ }^{2}$, Fariba Aghabaglou ${ }^{1}$, lan Ghanavati ${ }^{1}$, and \\ Ali Tamayol ${ }^{1, *}$ \\ ${ }^{1}$ Department of Mechanical and Materials Engineering, University of Nebraska, Lincoln, NE, \\ 68508, USA. \\ ${ }^{2}$ The University of Chicago Medical Scientist Training Program, Pritzker School of Medicine, \\ University of Chicago, Chicago, Illinois, USA.
}

\begin{abstract}
Chronic non-healing wounds are major healthcare challenges that affect a significant number of people; they exert a severe financial burden and are the leading cause of limb amputation.

Although chronic wounds are locked in a persisting inflamed state, they are dynamic and proper therapy requires identifying abnormalities, administering proper drugs and growth factors, and modulating the conditions of the environment. In this article, we will discuss technologies that have been developed to actively monitor the wound environment. We also highlight drug delivery tools that have been integrated with bandages to facilitate precise temporal and spatial control over drug release and review automated or semi-automated systems that can respond to the wound environment.
\end{abstract}

\section{Keywords}

Wound care; Smart bandages; Flexible electronics; Drug delivery; Integrated systems

\section{Wound biology and wound healing}

Chronic wounds have been estimated to affect 2-4.5 million people in the US and to cost the US economy $\$ 25$ billion per year. This burden is increasing due to rising healthcare cost, an aging population, and greater incidence of comorbidities such as diabetes [9]. Wound healing occurs through sequential and carefully synchronized biological events that are dysregulated in chronic wounds (Box 1). Various local and systemic factors can negatively affect successful wound healing, including infection, chronic inflammation, low nutrition content, increased local pressure, and poor perfusion [12]. To promote wound healing, the host's blood glucose and nutrition should be optimized [17]. The wound bed must be vascularized for proper oxygenation and nutrient delivery to the healing tissue [18].

\footnotetext{
"Corresponding author: A. Tamayol (900 N16th Street, Room NH W332, Lincoln, NE, 68508; Tel: (402) 472-1601, atamayol@unl.edu, Twitter: @lab_limb).

Publisher's Disclaimer: This is a PDF file of an unedited manuscript that has been accepted for publication. As a service to our customers we are providing this early version of the manuscript. The manuscript will undergo copyediting, typesetting, and review of the resulting proof before it is published in its final citable form. Please note that during the production process errors may be discovered which could affect the content, and all legal disclaimers that apply to the journal pertain.
} 
Bacterial load and exudate generation should also be minimized. Chronic wounds are at a high risk of bacterial infection as their hypoxic environment, rich with proteins, creates a suitable environment for bacterial growth [20]. Different pathogens usually reside in chronic wounds, but their presence cannot necessarily be interpreted as wound infection, as some studies support the presence of low levels of bacteria in the promotion of wound healing [23]. Bacteria have been found to produce proteolytic enzymes that facilitate the digestion of debris and stimulate protease release from neutrophils, however, beyond a critical colonization, infection occurs and wound healing can be impaired. [18]. Debridement is important since nonviable tissue prevents the movement of cells that is needed to build granulation tissue.

Wound healing technologies constitute a major commercial enterprise, with the market for products involved in wound closure exceeding $\$ 15$ billion and the market for skin scar prevention accounting for another $\$ 12$ billion [1]. The ideal wound care technology would 1) create a moist, clean, and warm environment, 2) protect the wound bed from mechanical trauma and bacterial infiltrations, 3) modulate exudate level, 4) allow for gas exchange, 5) promote thermal insulation, 6) be non-toxic and non-allergenic, and 7) deliver therapeutic compounds essential for healing with optimal temporal profile [26]. There have been various studies on the role of highly regulated factors involved in wound healing, such as inflammatory cells, cytokines, and chemokines in wound healings. In addition, significant progress has been made in the field of drug delivery to improve the bioavailability of administered drugs at the injury site. Wearable sensors have also been developed that can precisely measure the level of important markers in the wound environment. Although there is a major gap between the wound care products used in clinical practice and the advanced platforms developed by researchers, it is expected that the next generation of multifunctional "smart" dressings will lead to a paradigm shift in wound care. Such smart platforms can integrate different types of (bio)sensors for real-time monitoring and active treatment of the wound. In this review, we will discuss the advantages of smart dressings. In addition, we will highlight some of the sensors and active drug delivery systems that have been developed for wound care, along with automated systems that can both sense and respond to relevant wound parameters. There has been significant progress in the development of smart materials that can respond to the variations in the wound environment, such as upregulation of inflammatory markers, variation in ionic concentration, temperature, or changes in the environmental $\mathrm{pH}[8,28]$. However, these systems cannot be actively controlled. Smart materials of this nature have been recently reviewed elsewhere [8] and will not be discussed in this review.

\section{The need for smart bandages in wound care}

Chronic wounds have diverse etiologies and thus diverse signatures. Despite their molecular and clinical heterogeneity, chronic wounds can be divided into three main categories: venous leg ulcers (VLUs) (see Glossary), diabetic foot ulcers (DFUs), or pressure ulcers (PUs) [33]. A number of dressings have been developed that are indicated for specific types of chronic wounds based on a wound conditions, such as being dry or exuding, superficial or deep, and clean or infected [26]. These dressings are associated with various limitations (Box 2). As the dynamics of the wound healing process continue to be better understood, 
systems that actively control the spatial and temporal profile of drug release would be extremely beneficial for wound care treatment.

Despite advances in wound healing technologies, there is still a need for devices that can provide diagnostic information, combat infection, and effectively heal chronic wounds by intervening in dysfunctional healing processes $[35,36]$. Such systems could revolutionize the wound care practice and have profound effects on therapeutic outcomes. Smart systems, which allow for sensing, responding, reporting, or a combination of such functions, can address many of the challenges associated with wound healing, particularly for chronic wounds, and may allow for better wound management, improving clinical outcomes by means such as detecting infections in a timely manner or providing alerts for patients. Sensors can be combined with active drug delivery systems to autonomously respond to potential infection or hyper-inflammation. These integrated systems, which are summarized in the following sections, also have the potential to reduce healthcare costs for patients, hospitals, and insurance providers.

\section{Dressings with integrated sensors}

Current wound dressings are mainly designed to keep the injury site sealed and protected. Some of them release drugs or compounds that can prevent infection and help with faster healing. A key limitation of these dressings is their inability to provide information about the healing status and the conditions of the wound environment with regards to its $\mathrm{pH}$, bacterial loading, tissue oxygenation, and the level of inflammation. Sensors in the wound environment can provide important information that would expedite the decision making process in wound care. In addition, they can decrease the frequent changing of the wound dressing. There are also significant benefits in reduction of healthcare cost and time of hospitalization [37]. Chronic ulcers, lower infected acute wounds, and large full-thickness burns are the main variety of wounds that can be targeted by sensor technology [5]. A number of markers have been identified that provide information on important physiological processes such as vascularization and inflammation. In addition, another important condition that can prevent healing of wounds is infection, which if not treated at the early stages can result in formation of established biofilms that are tough to treat. Once biofilm is established in a necrotic tissue, surgical debridement and limb amputation are required. As a result, infected DFUs are the dominant cause of non-traumatic lower-extremity limb amputation [38]. Sensors intended for use in wound care products should have specific properties, such as having suitable flexibility to conform to body contours, being non-toxic and immune compatible, and being resistant to wound exudate, which has a varying $\mathrm{pH}$ and is rich with proteins and enzymes. Researchers have developed a number of sensors that can measure important biomarkers in the wound environment (Table 1). In this section, we will review dressings engineered with integrated sensors.

\section{Dressings with $\mathrm{pH}$ sensors}

Various types of $\mathrm{pH}$ sensors have been fabricated for wound care applications. For example, several types of electrochemical $\mathrm{pH}$ sensors have been developed that can continuously monitor the wound environment. Electrochemical $\mathrm{pH}$ sensors usually utilize potentiometric 
measurement, conducting polymer $\mathrm{pH}$ sensors [50, 51], metal oxide [52], ion-selective electrodes [53], and ion-selective field effect transistors [54]. Potentiometric measurements have been used in engineering a number of wound dressings with integrated sensors. These sensors can be fabricated on stretchable, flexible substrates. In a notable study, a low-cost stretchable sensor was created by spraying conductive inks and polymers on ecoflex substrate [39]. The sensor had a Nernstian response to changes in $\mathrm{pH}$ response and showed reproducible data upon cyclic changes in the environmental $\mathrm{pH}$. The sensor could form and maintain conformal contact with curved surfaces (Figure $1 \mathrm{a}-\mathrm{c}$ ).

Colorimetric sensors are another class of $\mathrm{pH}$ sensors that can be used to engineer bandages. These sensors are robust, easy-to-use, and can be utilized without integrated electronics. The readout of these sensors is usually based on image processing, although if the color change is sufficiently vivid, a naked eye view can be used for estimation of $\mathrm{pH}$ value or identification of wound status. However, a key challenge associated with the utilization of luminescence systems is protecting the skin from the leached dye. In one attempt to combat this challenge, $\mathrm{pH}$-responsive dyes were incorporated in mesoporous silica particles (MSPs) to prevent leakage during their use. The particles were then incorporated into flexible hydrogel fibers fabricated through microfluidic spinning. The fibers were then attached to a transparent medical tape for long-term monitoring of cutaneous wounds with the possibility of directly printing onto the medical tape (Figure $1 \mathrm{~d}-\mathrm{f}$ ) [40]. The $\mathrm{pH}$ of the wound was determined by processing images captured using smartphones. Image-based data analysis is susceptible to errors introduced due to lighting and image quality, so a hydrogel dressing was fabricated to solve this challenge where the hydrogel carried $\mathrm{pH}$-responsive dyes. $\mathrm{pH}$ values were measured using an integrated photodiode that could wirelessly communicate with smartphones [55]. Because integrating readout platforms or image analysis can be challenging, the idea of using dyes which their color change are visible to naked eye has been pursued. In one example, a dye was developed that could be loaded into wound dressings and would illuminate different colors upon exposure to UV light to predict the wound $\mathrm{pH}$ level [41].

\section{Temperature sensors}

Similar to $\mathrm{pH}$ sensors, flexible colorimetric and electrochemical temperature sensors have been widely used in biomedical application [43]. Microfabricated metallic resistive sensors are the most common type of flexible temperature sensors. One example dressing was fabricated from microfabricated arrays of temperature sensors for skin temperature mapping of cutaneous wounds [44]. The conductive lines were stretchable and were fabricated on a flexible substrate and could form a conformal contact to the skin (Figure $2 \mathrm{a}-\mathrm{d}$ ). The platform was integrated and could provide a map of temperature distribution in the wound area [44]. To reduce the cost of fabrication, material conductive inks have also been used for the fabrication of these sensors. One key challenge associated with conductive inks is the change in their electrical resistance due to mechanical strain that can interfere with the accuracy of the temperature sensors. However, using suitable porous substrates can alleviate this issue significantly. In one example, a nanofibrous substrate was used to fabricate flexible sensors based on silver inks (Figure $2 \mathrm{e}-\mathrm{g}$ ) [42]. The results showed a relatively linear response in temperature variations in the range of $25^{\circ} \mathrm{C}-35^{\circ} \mathrm{C}$. In another study, a 
nanocomposite of poly(styrene-b-(ethylene-co-butylene)-b-styrene) and multiwall carbon nanotubes was fabricated and used to engineer a dressing for monitoring DFUs and VLUs [56]. One of the factors that affects the accuracy of the sensor readings is its conformal contact with the skin. To adhere sensors to skin long-term, octopus-mimicking surfaces were developed and utilized as a substrate for fabrication of printed carbon-based sensors (Figure $2 \mathrm{~h}-\mathrm{j}$ ) [57]. In general, using carbon nanotube-based materials to fabricate sensors has enabled engineering low-cost devices with high sensitivity. However, the use of carbon nanotubes in biomedical engineering has been controversial due to their potential toxicity [58].

\section{Oxygen sensors}

Chronic wounds have insufficient wound oxygenation due to inefficient vascularization. As a consequence, acute hypoxia in a chronic wound may be detrimental to the healing process and result in unnecessary tissue loss [59]. Hence, monitoring tissue oxygenation provides valuable data on wound healing. One study developed a flexible and wireless smart bandage with a customized oxygen sensor from off-the-shelf electronic parts to monitor the wound bed in real time [46]. The oxygen sensor was structured using an electrochemical galvanic cell on a flexible parylene-C substrate. In this platform, silver and electroplated zinc electrodes worked as the cathode and anode, respectively. This system was tested on a simulated wound testing setup.

Another study developed a colorimetric sensor that could be painted on the surface of the wound in order to form a thin and stable layer with conformal contact, allowing for oxygen level monitoring (Figure $2 \mathrm{k}-\mathrm{m}$ ) [45]. This platform utilized a reference dye and porphyrindendrimer phosphor, which fluoresced in the presence of oxygen. The platform was successfully tested both ex vivo and in vivo to monitor the progression of burns and integration of skin grafts and to sense tissue ischemia. A key challenge in designing oxygen sensors is distinguishing the tissue oxygen level from the oxygen introduced topically from air. These sensors usually use an oxygen-impermeable coating to address this challenge, which in turn can negatively affect the wound healing.

\section{Moisture sensors}

In a notable study, electrochemical sensors were used underneath dressings in order to monitor the level of moisture at the time of bandage change [47]. The results showed that more than $40 \%$ of dressing changes occurred before the optimal time, which indicated the need to improve current clinical protocols.

\section{Mechanical and electrical sensors}

The mechanical and electrical properties of skin change in response to skin disorders or diseases. In addition, the mechanical and electrical stimulation of a wound has been proven to facilitate wound healing and prevent infection. Thus, sensors that can monitor the stiffness and impedance of the skin or wound environment can provide important data on the tissue conditions. In a notable study, impedance spectroscopy using flexible electrode arrays was used to predict the skin condition and onset of skin damage in response to excessive pressure. The results showed the suitability of the approach for predicting PUs [48]. In a 
study on a rat model, the sensor couple map the impedance magnitude, phase angle and damage threshold, which otherwise could not be detected with a naked-eye inspection. Another study developed a flexible fabric-based electrical pressure sensor that could map the applied pressure on skin for assessment of the chance of formation or deterioration of decubitus ulcers [60]. The sensor array wirelessly could communicate with external devices. In one other study, a flexible strain sensor was fabricated on elastic biodegradable substrates suitable for use as wound dressings [42]. The sensor operated based on measuring varying magnetic flux through a known area, which occurred when the sensor stretched. Such sensors could provide information on patients' movements and their potential risk in preventing wound healing.

In general, wound care products equipped with sensing capabilities can significantly change the current clinical practice of wound treatment. The generated information can provide valuable insight on the status of the wound, which is especially important in the case of diabetic wounds, where regular signs of abnormality and infection such as redness, swelling, and hyperthermia are not as severe as those in regular wounds. Thus, current clinical approaches used for wound inspection are usually insufficient; the engineered smart bandages reviewed here could bolster current clinical practices, given that they can actively extract information about the wound environment. Currently, however, the number of markers that are being monitored are limited and the majority of the markers are nonspecific. Thus, there is a need to identify more suitable markers as well as designing platforms that integrate multiple types of sensors. A recent study reported different threadbased sensors for medical diagnostics that could measure $\mathrm{pH}$ level, glucose, temperature, and strain of a targeted tissue [61]. Similar platforms could substantially enhance the impact of these smart bandages. Beyond this, flexibility and longevity of currently engineered sensors are not ideal for wound care applications and require improvement. One of the key limitations of sensors for wound care is their durability, as the wound environment is moist and concentrated with various proteins which can affect the sensitivity of the sensors in a short period of time.

\section{Wound dressings with active drug release}

Significant progress has been made in identifying biological factors or drugs that modulate one or multiple physiological processes involved in tissue healing. The proper delivery of these factors to the wound bed has been the subject of numerous studies, which have been recently reviewed [8]. Technologies used in drug delivery are considered either passive or active, depending on the ability to externally control the drug release profile or permeation rate into the tissue [62]. Passive methods refer to systems with controlled release of compounds from dressings or structures, drug carriers, and scaffolding materials [8, 63]. Such methods are relatively easy-to-integrate within wound dressings and can be used to deliver different compounds with predesigned dosages. The properties of the wound environment as well as the physiological processes dynamically change during the healing process of wounds. As a result, the need for active compounds could be different at different healing stages and time points. Although passive drug delivery methods are simple and effective in the delivery of therapeutics, they are incapable of adjusting the release profiles on the fly. There is another class of semi-passive drug delivery systems that utilize smart 
materials to respond to variations in their environment and could adjust the drug release rate based on the properties of the environment. These materials typically respond to changes in the wound $\mathrm{pH}$, temperature, and enzyme level and do not allow externally active control over the drug release profile. These systems have recently been reviewed [8] and are not discussed here.

Active drug delivery systems, on the other hand, enable the precise control of temporal or even spatial release profile. These systems are generally designed to only release drugs in response to an external stimulation that drives the drug toward the wound bed. Recent advances in wearable technology and microfabrication have enabled the development of such devices. Several different mechanisms have been used to engineer active drug delivery tools for wound care, such as mechanical and piezoelectric pumping, thermally actuating thermo-responsive systems, iontophoretic transport of compounds, and active jet injection. In pump-based systems, the generated pressure or induced flow moves the drug or drug carriers towards the point of delivery. Thermally actuating systems utilizes an external heater to heat up a thermo-responsive material, which in turn releases the drug into the environment. In iontophoresis, the movement of ions across a membrane is enhanced using an externally applied electrical potential difference. These systems are usually used to transfer charged molecules or drug carriers. In jet injectors, a high-velocity jet opens pores in the skin and facilitates the transfer of molecules transdermally. However, jet-based systems are bulky and are difficult to integrate into wearable systems. All of these solutions (except jet injectors) have been integrated in wound dressings to enable active drug delivery.

Pumping is probably the most conventional way of delivering liquids containing drugs or drug carriers into the wound bed. Miniaturized pumps have been microfabricated that allow the transport of minute amounts of solutions and have also been widely utilized in drug delivery. These pumping systems have also been integrated with dressings to enable active delivery of compounds to the wound area. In one example, a wearable dressing was developed in which a manual pump was used to flow $\mathrm{H}_{2} \mathrm{O}_{2}$ into a microchannel array over a porous membrane coated with catalyst particles [64]. $\mathrm{H}_{2} \mathrm{O}_{2}$ was dissociated in water and oxygen, where oxygen was permeating towards the wound bed. Micropumps have also been integrated in skin patches with hollow microneedles to transdermally inject precise volumes of therapeutics such as insulin. In one example, microneedles were used for a continuous subcutaneous infusion drug delivery system to administer morphine sulfate. The delivery rate of morphine was controlled by using an integrated micro-infusion pump [65].

Thermal stimulation has been one of the most popular approaches for triggering drug release in wound care. Thermal stimulation is generally considered to be safe and in some cases the localized increase of temperature has resulted in faster wound healing. Localized increase in skin temperature can also enhance the skin permeability facilitating drug penetration [66]. One of the more prominent examples of thermally actuated wound dressings was proposed by Bagherifard and colleagues [67]. In this platform, thermo-responsive particles of $\mathbf{p o l y}(\mathbf{N}$ isopropylacrylamide) (pNIPAM) were fabricated using a microfluidic system. The particles were loaded with the drug and were placed in a flexible hydrogel sheet (Figure $3 a, b)$. The hydrogel was attached to a dressing with an integrated flexible heater [67]. In another study, thermo-responsive chitosan-based nanoparticles of less than $100 \mathrm{~nm}$ in 
diameter were fabricated and mixed with a polymeric solution [68]. The mixture was then electrospun to form flexible nanofibrous substrates, where the nanoparticles were embedded within the nanofibers (Figure 3c-e). A flexible and biodegradable heater was sputtered on the substrate to create an integrated dressing. The platform had a close to zero passive release and the release rate could be controlled by adjusting the substrate temperature [68].

Wound healing involves multiple physiological processes and the occurrence of chronic and non-healing wounds is multifactorial. As a result, the ability to release multiple drugs with independent release profiles and quantities is important for superior treatment of chronic wounds. Although passive release systems have been developed that are able to release drugs with different profiles, none of these systems have allowed for active control over the release profiles. Recently, a textile based dressing was fabricated using composite fibers with a core electrical heater covered by a layer of hydrogel containing thermo-responsive drug carriers of poly(ethylene glycol diacrylate) PEGDA-pNIPAM (Figure 3f-i) [69]. Each thread was composed of a conductive core thread serving as a flexible microheater which was coated with a PEGDA- Alg hydrogel carrying thermo-responsive drug carriers. Each fiber was connected to a microcontroller that was connected to a smartphone and could be independently triggered to release a specific dosage of the encapsulated drug in that fiber. The benefit of releasing multiple drugs was demonstrated in a culture of infected endothelial cells. In addition, the release of vascular endothelial growth factor (VEGF) from the bandage facilitated the healing of wounds in diabetic mice [69].

Iontophoresis is a well-established technology for transdermal delivery of compounds. Wearable iontophoretic drug delivery systems have already been fabricated and integrated with bandages and skin patches [70]. Similar to thermal stimulation, electrical stimulation has also been shown to positively impact the wound healing [71]. Iontophoretic drug delivery platforms, however, do not suffer from the risk of self-trigger due to changes in environmental temperature, which makes them more suitable for wound healing applications. In addition, these systems usually deliver therapeutics to deeper layers of skin and can enhance the drug concentration close to the healing tissue. The application of these platforms for engineering smart bandages that enable active control over the drug release kinetic are limited to a few examples. In one example, the benefit of electrical stimulation combined with the iontophoretic administration of zinc sulfate in the healing of abdominal surgical wounds in both diabetic and non-diabetic rats was demonstrated [72]. In another study, the iontophoretic delivery of nitric oxide resulted in enhanced free flap survival in comparison to its delivery using hypodermic needles [73].

Smart bandages capable of actively delivering therapeutics are important as they enable the planning of a treatment that consists of releasing drugs at the right time with the right dosage. Among different external actuation mechanisms, pumping is probably the easiest method. However, for engineering bandages micropumps are required that can be integrated in a wearable platform and deliver minute amounts of solutions. As pump-based systems usually transfer aqueous solutions, hydrophobic drugs should be encapsulated within drug carriers that can be dispersed in aqueous solutions. Thermo-responsive drug delivery platforms have shown great promise, however, they do not offer a zero release at room temperature and they are susceptible to self-triggering in warm weather. Iontophoretic 
platforms are also usually limited to hydrophilic drugs unless encapsulated in proper drug carriers. If these platforms can be designed to enable the independent release of different drugs, however, they may prove to be superior to other platforms.

\section{Automated wound dressings}

The majority of existing medical devices are either equipped with sensors or actuators that can deliver therapies. In recent years, along with the advancement of flexible electronics and packaging, multifunctional medical devices have been developed that can both sense and deliver therapeutics. In the field of wound care, the complexity of the physiological events has made the decision making process extremely challenging. As a result, there has been a preference towards leaving the decision making process to medical professionals. However, the recent progress in the field of wound biology has improved our understanding of physiological processes. This progress, combined with the realization of miniaturized data processing systems and controllers, has enabled researchers to design systems that can sense and interpret data and decide on the delivery of therapeutics.

A key challenge in designing automated dressings is the identification of suitable parameters to be monitored and pathophysiological processes to modulate. There have been a number of markers that have been related to (patho)physiological processes. For instance, tissue oxygenation has been considered as a measure of vascularization, while inflammation and infection have been identified by varying wound temperature, $\mathrm{pH}$, and enzyme levels. The two pathophysiological processes whose modulation can benefit from automated systems are inflammation and infection. While inflammation is essential for healing, as it helps with removal of debris and dead tissue, hyper-inflammation should be avoided. Infection is one of the major pathophysiological challenges that prevents wound healing and necessitates surgical intervention. Although the delivery of antibiotics at early stages of colonization is more effective, the prophylactic use of antibiotics has shown limited success, can retard the healing rate, and can lead to the formation of antibiotic resistance [8]. Thus, antibiotics should be used when needed to eliminate the side effects associated with infection or antibiotic overuse.

Among different biological markers, $\mathrm{pH}$ has been widely used to design automated wound dressings. There are only a few examples of automated dressings published in the literature (Figure 4). A fully automated wound dressing was recently engineered that benefited from an electrochemical sensor that could measure the $\mathrm{pH}$ of the wound (Figure 4a) [74]. Data was processed by an on-board controller to detect potential $\mathrm{pH}$ values indicating infection. In this case, the controller could automatically trigger the integrated flexible heater in order to heat the hydrogel that carried drug carriers, which could then release antibiotics. The effectiveness of the platform in treating infection was demonstrated in vitro (Figure $4 b-d$ ) [74]. In another recent study, a hydrogel-based dressing could continuously monitor wound $\mathrm{pH}$, monitor for infection, and deliver antibiotics into the wound bed, if necessary [75]. This semi-automated platform utilized an immobilized $\mathrm{pH}$ sensitive dye that could be imaged using a smartphone to determine potential infection (Figure $4 \mathrm{e}, \mathrm{f}$ ). The dressing also carried thermo-responsive drug carriers that could be triggered using a smartphone app to release antibiotics upon infection [75]. 
Automated dressings can revolutionize wound care, significantly improve the effectiveness of therapies, and enhance a patient's comfort. However, there is a need to engineer dressings that can integrate sensors to detect more specific markers for better interpretation of biological events. In addition, the ability of releasing multiple drugs with different release profiles could enhance the effectiveness of such platforms.

\section{Discussions, concluding remarks, and future outlook}

Chronic wounds remain a major concern of the healthcare system. With a growing number of diabetic patients, the number of diabetic wounds and ulcers will likely increase. Burns, PUs, and other wounds are also expected to grow, especially in elderly populations. The changes in living standards in developed countries have affected healthcare products and their goals. Currently, tools and devices for cosmetic applications are becoming more important as scarring and some other skin disorders such as keloids can exert psychological discomfort. Thus, there have been substantial efforts in developing more effective therapeutics to treat different types of wounds. These efforts have resulted in the identification of growth factors, proteins, and drugs that can improve or modulate physiological processes that affect wound healing. In addition, various drug delivery strategies have been developed and mastered to more effectively deliver the therapeutics to the wound bed. However, recent developments in the field of flexible and wearable electronics has enabled the generation of a new class of dressings that can actively help with understanding the wound condition or the interference of the tissue healing. Although these dressings provide invaluable information about the wound status, they do not target specific markers. One area that can substantially improve the field is the development of wearable biosensors that can detect proteins and antigens in the wound bed. Considering that infection is a key challenge in wound care and can even lead to life-threatening conditions, sensors that can directly measure the bacterial loading would be extremely valuable.

The wound environment is rich with cytokines and proteins that play roles in various physiological processes. As a result, the concentration of the biomarkers could provide important information on the rate of salient physiological processes. Despite the importance of monitoring biomarkers, biosensors are sufficiently complex that there is no bandage that can detect the concentration of a particular protein. One class of biomarkers that can be detected using conventional electrochemical systems is enzymes [55]. A group of enzymes that have attracted noticeable attention are neutrophil secretomes such as elastase and cathepsins. Chronic wounds suffer from excessive inflammation and, once infected, from hyper-inflammation. Recent biological and clinical observations have shown that the neutrophils' secretomes are an excellent biological marker for the detection of infection [76]. In fact, pathogens have the ability to hijack the immune system, and in particular neutrophils, in order to induce hyper-inflammation and the production of exudates that can become an excellent source of nutrients for pathogens and biofilms to rapidly grow. Cathepsin and elastase are among these pro-inflammatory markers and there have been a number of studies that have confirmed the correlation between their levels and infection [49]. The use of photoactive materials that can report the level of various markers can potentially facilitate the development of advanced biosensors for wound care. We expect that by engineering initially wearable enzymatic sensors and later protein detection systems that 
can be integrated into wound dressings, wound care practice would substantially be improved. In this case, by having a clear vision of the wound environment, the suitable treatment could be administered.

Wearable electronics and devices have also been used to deliver drugs and factors to the wound bed with a more controlled profile. Since the wound environment is dynamic and the rate of physiological processes varies at different healing stages, the ability to actively control the release of drugs and factors can result in faster healing. However, the majority of these systems suffer from passive release and this passive release can lead to complications. For example, if bacteria are exposed to an insufficient concentration of antibiotics, they can develop resistance against the antibiotic, which is a major healthcare problem. In addition, chronic wounds exude and are covered with dead or necrotic tissues. Thus, the development of drug delivery tools that can facilitate the delivery of therapeutics across these barriers into the healing tissue is expected to have a significant impact on the therapy outcome.

The emergence of automated bandages and telemedicine is expected to change clinical practice especially in remote areas. In the field of wound care, more automated dressings that can sense and deliver therapeutics automatically or semi-automatically would significantly improve a patient's comfort and reduce the complications associated with these wounds. However, better power sources, stable sensors, and reliable networks for data management are all required, rely on multifaceted research activities, and have yet to be developed (see Outstanding Questions).

\section{Acknowledgements}

The authors declare no conflict of interests in this work. This research has been partially supported by National Institutes of Health (GM126831), the University of Nebraska-Lincoln and the Nebraska Tobacco Settlement Biomedical Research Enhancement Funds.

\section{Glossary}

\section{Diabetic foot ulcers (DFUs)}

non-healing full-thickness wounds that extend through the dermis, below the ankle and are often caused by repetitive injury to the site. DFUs are common complications of poorly controlled diabetes

\section{Mesoporous silica particles (MSPs)}

materials with that have pore sizes and surface areas in the range of 2-50 nm, high surface free energy, and applications in adsorption, catalysis, separation, and drug delivery

\section{poly(N-isopropylacrylamide) (pNIPAM)}

a thermo-responsive polymer that is soluble at room temperature but phase separates at body temperature

\section{poly(ethylene glycol diacrylate) (PEGDA)}

a hydrophilic, elastic hydrogel that gels rapidly at room temperature in the presence of photo-initiator and UV light 


\section{Pressure ulcers (PUs)}

injuries to the skin and underlying tissue due to prolonged pressure on the skin. Pressure ulcers commonly occur on skin over bony areas of the body, such as the hips, heels, tailbone, and ankles

\section{Vascular endothelial growth factor (VEGF)}

a potent angiogenic factor that is involved in various physiological functions such as hematopoiesis, wound healing, development, and bone formation

\section{Venous leg ulcers (VLUs)}

open lesions that occur between the ankle joint and the knee in patients with venous disease. These ulcers occur in advanced forms of chronic venous disorders like varicose veins and lipodermatosclerosis

\section{References}

1. Han G and Ceilley R (2017) Chronic Wound Healing: A Review of Current Management and Treatments. Adv Ther 34 (3), 599-610. [PubMed: 28108895]

2. Negar F et al. (2018) Patient-Specific Bioinks for 3D Bioprinting of Tissue Engineering Scaffolds. Advanced Healthcare Materials 7 (11), 1701347.

3. Jones V et al. (2006) ABC of wound healing: wound dressings. BMJ: British Medical Journal 332 (7544), 777. [PubMed: 16575081]

4. Serra MB et al. (2017) From Inflammation to Current and Alternative Therapies Involved in Wound Healing. Int J Inflam 2017, 3406215. [PubMed: 28811953]

5. Dargaville TR et al. (2013) Sensors and imaging for wound healing: a review. Biosens Bioelectron 41, 30-42. [PubMed: 23058663]

6. Liu Y et al. (2002) Fibroblast proliferation due to exposure to a platelet concentrate in vitro is $\mathrm{pH}$ dependent. Wound Repair Regen 10 (5), 336-40. [PubMed: 12406171]

7. Schneider LA et al. (2007) Influence of $\mathrm{pH}$ on wound-healing: a new perspective for woundtherapy? Arch Dermatol Res 298 (9), 413-20. [PubMed: 17091276]

8. Saghazadeh S et al. (2018) Drug delivery systems and materials for wound healing applications. Advanced Drug Delivery Reviews.

9. Sen CK et al. (2009) Human skin wounds: a major and snowballing threat to public health and the economy. Wound Repair Regen 17 (6), 763-71. [PubMed: 19903300]

10. Darby IA et al. (2014) Fibroblasts and myofibroblasts in wound healing. Clin Cosmet Investig Dermatol 7, 301-11.

11. Leveen $\mathrm{HH}$ et al. (1973) Chemical acidification of wounds. An adjuvant to healing and the unfavorable action of alkalinity and ammonia. Ann Surg 178 (6), 745-53. [PubMed: 4759406]

12. Guo S and Dipietro LA (2010) Factors affecting wound healing. Journal of dental research 89 (3), 219-29. [PubMed: 20139336]

13. Mayet $\mathrm{N}$ et al. (2014) A comprehensive review of advanced biopolymeric wound healing systems. J Pharm Sci 103 (8), 2211-30. [PubMed: 24985412]

14. Dowsett $\mathrm{C}$ et al. (2014) Reconciling increasing wound care demands with available resources. J Wound Care 23 (11), 552, 554, 556-8 passim. [PubMed: 25375403]

15. Moore K et al. (2006) Prediction and monitoring the therapeutic response of chronic dermal wounds. Int Wound J 3 (2), 89-96. [PubMed: 17007340]

16. Jones EM et al. (2015) The Effect of $\mathrm{pH}$ on the Extracellular Matrix and Biofilms. Adv Wound Care (New Rochelle) 4 (7), 431-439. [PubMed: 26155386]

17. Williams JZ and Barbul A (2003) Nutrition and wound healing. Surg Clin North Am 83 (3), $571-$ 96. [PubMed: 12822727] 
18. Sood A et al. (2014) Wound Dressings and Comparative Effectiveness Data. Adv Wound Care (New Rochelle) 3 (8), 511-529. [PubMed: 25126472]

19. Khan AA et al. (2004) Topical radiant heating in wound healing: an experimental study in a donor site wound model*. Int Wound J 1 (4), 233-40. [PubMed: 16722872]

20. Liu YC et al. (2011) Does inflammation have a role in the pathogenesis of venous ulcers? A critical review of the evidence. The Journal of investigative dermatology 131 (4), 818-27. [PubMed: 21307876]

21. Nakagami G PhD, et al. (2010) Predicting delayed pressure ulcer healing using thermography: a prospective cohort study. Journal of Wound Care 19 (11), 465-472. [PubMed: 21135794]

22. Martinez-Jimenez MA et al. (2013) Local use of insulin in wounds of diabetic patients: higher temperature, fibrosis, and angiogenesis. Plast Reconstr Surg 132 (6), 1015e-9e.

23. Ovington L (2003) Bacterial toxins and wound healing. Ostomy/wound management 49 (7A Suppl), 8-12.

24. Cuthbertson DP and Tilstone WJ (1967) Effect of environmental temperature on the closure of full thickness skin wounds in the rat. Q J Exp Physiol Cogn Med Sci 52 (3), 249-57. [PubMed: 5182406]

25. Kurz A et al. (1996) Perioperative normothermia to reduce the incidence of surgical-wound infection and shorten hospitalization. Study of Wound Infection and Temperature Group. N Engl J Med 334 (19), 1209-15. [PubMed: 8606715]

26. Dabiri G et al. (2016) Choosing a Wound Dressing Based on Common Wound Characteristics. Adv Wound Care (New Rochelle) 5 (1), 32-41. [PubMed: 26858913]

27. Schreml S et al. (2010) Oxygen in acute and chronic wound healing. Br J Dermatol 163 (2), $257-$ 68. [PubMed: 20394633]

28. Huang $X$ et al. (2015) A self-regulating antimicrobial model based on the ion-exchange stimuli. Journal of Materials Science: Materials in Medicine 26 (7), 208. [PubMed: 26159674]

29. Semenza GL (2010) Vascular responses to hypoxia and ischemia. Arterioscler Thromb Vasc Biol 30 (4), 648-52. [PubMed: 19729615]

30. Ruangsetakit $\mathrm{C}$ et al. (2010) Transcutaneous oxygen tension: a useful predictor of ulcer healing in critical limb ischaemia. Journal of wound care 19 (5), 202-206. [PubMed: 20505593]

31. Cutting KF (2003) Wound exudate: composition and functions. Br J Community Nurs 8 (9 Suppl), suppl 4-9.

32. Harding K (2007) Diagnostics and wounds. World Union Wound Heal. Soc. WUWHS Consens. Doc

33. Nunan R et al. (2014) Clinical challenges of chronic wounds: searching for an optimal animal model to recapitulate their complexity. Disease models \& mechanisms 7 (11), 1205-13. [PubMed: 25359790]

34. Dyson M et al. (1992) Comparison of the effects of moist and dry conditions on the process of angiogenesis during dermal repair. J Invest Dermatol 99 (6), 729-33. [PubMed: 1281862]

35. Mast BA and Schultz GS (1996) Interactions of cytokines, growth factors, and proteases in acute and chronic wounds. Wound Repair Regen 4 (4), 411-20. [PubMed: 17309691]

36. Palecek E et al. (2002) New approaches in the development of DNA sensors: hybridization and electrochemical detection of DNA and RNA at two different surfaces. Bioelectrochemistry 56 (12), 85-90. [PubMed: 12009450]

37. Mehmood N et al. (2014) Applications of modern sensors and wireless technology in effective wound management. J Biomed Mater Res B Appl Biomater 102 (4), 885-95. [PubMed: 24142514]

38. Guo J et al. (2017) Meta-analysis on the treatment of diabetic foot ulcers with autologous stem cells. Stem Cell Research \& Therapy 8, 228. [PubMed: 29037219]

39. Rahimi R et al. (2017) Highly Stretchable Potentiometric pH Sensor Fabricated via Laser Carbonization and Machining of Carbon-Polyaniline Composite. ACS Applied Material Interfaces 9 (10), 9015-9023.

40. Tamayol A et al. (2016) Flexible pH-Sensing Hydrogel Fibers for Epidermal Applications. Advanced Healthcare Materials 5 (6), 711-9. [PubMed: 26799457] 
41. Panzarasa G et al. (2017) The pyranine-benzalkonium ion pair: A promising fluorescent system for the ratiometric detection of wound $\mathrm{pH}$. Sensors and Actuators B: Chemical 249, 156-160.

42. Hassani NA et al. (2014) Biodegradable nanofibrous polymeric substrates for generating elastic and flexible electronics. Advanced Materials 26 (33), 5823-5830. [PubMed: 25044366]

43. Trung TQ et al. (2016) An All- Elastomeric Transparent and Stretchable Temperature Sensor for Body- Attachable Wearable Electronics. Advanced Materials 28 (3), 502-509. [PubMed: 26607674]

44. Hattori Y et al. (2014) Multifunctional skin-like electronics for quantitative, clinical monitoring of cutaneous wound healing. Adv Healthc Mater 3 (10), 1597-607. [PubMed: 24668927]

45. Li Z et al. (2014) Non-invasive transdermal two-dimensional mapping of cutaneous oxygenation with a rapid-drying liquid bandage. Biomed Opt Express 5 (11), 3748-64. [PubMed: 25426308]

46. Mostafalu P et al. (2015) Wireless Flexible Smart Bandage for Continuous Monitoring of Wound Oxygenation. IEEE Trans Biomed Circuits Syst 9 (5), 670-7. [PubMed: 26552096]

47. Milne SD et al. (2016) A wearable wound moisture sensor as an indicator for wound dressing change: an observational study of wound moisture and status. International Wound Journal 13 (6), 1309-1314. [PubMed: 26561281]

48. Swisher SL et al. (2015) Impedance sensing device enables early detection of pressure ulcers in vivo. Nat Commun 6, 6575. [PubMed: 25779688]

49. Wignarajah S et al. (2015) Colorimetric Assay for the Detection of Typical Biomarkers for Periodontitis Using a Magnetic Nanoparticle Biosensor. Analytical Chemistry 87 (24), 1216112168. [PubMed: 26631371]

50. Artukovic E et al. (2005) Transparent and flexible carbon nanotube transistors. Nano Lett 5 (4), 757-60. [PubMed: 15826123]

51. Adhikari B and Majumdar S (2004) Polymers in sensor applications. Progress in Polymer Science 29 (7), 699-766.

52. Park S et al. (2005) pH-sensitive solid-state electrode based on electrodeposited nanoporous platinum. Anal Chem 77 (23), 7695-701. [PubMed: 16316178]

53. Bakker E and Pretsch E (2002) The new wave of ion-selective. Anal Chem 74 (15), 420A-426A. [PubMed: 11811417]

54. Bergveld P Thirty years of ISFETOLOGY: What happened in the past 30 years and what may happen in the next 30 years. Sens. Actuators B Chem. 88 (1), 1-20.

55. Kassal $P$ et al. (2015) Smart bandage with wireless connectivity for uric acid biosensing as an indicator of wound status. Electrochemistry Communications 56, 6-10.

56. Salvo P et al. (2017) Temperature-and pH-sensitive wearable materials for monitoring foot ulcers. International Journal of Nanomedicine 12, 949. [PubMed: 28203074]

57. Oh JH et al. (2018) Fabrication of High-Sensitivity Skin-Attachable Temperature Sensors with Bioinspired Microstructured Adhesive. ACS Appl Mater Interfaces 10 (8), 7263-7270. [PubMed: 29400434]

58. Sanvicens N and Marco MP (2008) Multifunctional nanoparticles - properties and prospects for their use in human medicine. Trends in Biotechnology 26 (8), 425-433. [PubMed: 18514941]

59. Sen CK (2009) Wound healing essentials: let there be oxygen. Wound Repair Regen 17 (1), 1-18. [PubMed: 19152646]

60. Chung P et al. (2013) Fabric-based pressure sensor array for decubitus ulcer monitoring. Conf Proc IEEE Eng Med Biol Soc 2013, 6506-9. [PubMed: 24111232]

61. Mostafalu P et al. (2016) A toolkit of thread-based microfluidics, sensors, and electronics for 3D tissue embedding for medical diagnostics. Microsystems \& Nanoengineering 2, 16039. [PubMed: 31057832]

62. Khademhosseini A et al. (2006) Microscale technologies for tissue engineering and biology. Proc Natl Acad Sci U S A 103 (8), 2480-7. [PubMed: 16477028]

63. Schreier H and Bouwstra J (1994) Liposomes and niosomes as topical drug carriers: dermal and transdermal drug delivery. J. Controlled Release 30 (1), 1-15.

64. Ochoa $\mathrm{M}$ et al. (2014) A paper-based oxygen generating platform with spatially defined catalytic regions. Sensors and Actuators B: Chemical 198, 472-478. 
65. Mikkelsen Lynch P et al. (2000) A pharmacokinetic and tolerability evaluation of two continuous subcutaneous infusion systems compared to an oral controlled-release morphine. J Pain Symptom Manage 19 (5), 348-56. [PubMed: 10869875]

66. Prausnitz MR et al. (2004) Current status and future potential of transdermal drug delivery. Nat Rev Drug Discov 3 (2), 115-24. [PubMed: 15040576]

67. Bagherifard S et al. (2016) Dermal Patch with Integrated Flexible Heater for on Demand Drug Delivery. Advanced Healthcare Materials 5 (1), 175-184. [PubMed: 26501166]

68. Tamayol A et al. (2017) Biodegradable elastic nanofibrous platforms with integrated flexible heaters for on-demand drug delivery. Scientific Reports 7 (1), 9220. [PubMed: 28835675]

69. Mostafalu P et al. (2017) A Textile Dressing for Temporal and Dosage Controlled Drug Delivery. Advanced Functional Materials 27 (41), 1702399.

70. Emaminejad S et al. (2017) Autonomous sweat extraction and analysis applied to cystic fibrosis and glucose monitoring using a fully integrated wearable platform. Proceedings of the National Academy of Sciences, 201701740.

71. Kloth LC (2005) Electrical stimulation for wound healing: a review of evidence from in vitro studies, animal experiments, and clinical trials. The international journal of lower extremity wounds 4 (1), 23-44. [PubMed: 15860450]

72. Langoni Cassettari L et al. (2014) Continuous Electrical Current and Zinc Sulphate Administered by Transdermal Iontophoresis Improves Skin Healing in Diabetic Rats Induced by Alloxan: Morphological and Ultrastructural Analysis. Journal of Diabetes Research 2014, 980232. [PubMed: 25254221]

73. Russell JA et al. (2010) Iontophoretic delivery of a nitric oxide donor improves local skin flap viability. Journal of Rehabilitation Research and Development 47 (1), 61-66. [PubMed: 20437327]

74. Mostafalu P et al. (2018) Smart bandage for monitoring and treatment of chronic wounds. Small In press.

75. Mirani B et al. (2017) An Advanced Multifunctional Hydrogel-Based Dressing for Wound Monitoring and Drug Delivery. Advanced Healthcare Materials 6 (19).

76. Hasmann A et al. (2011) Sensor materials for the detection of human neutrophil elastase and cathepsin $\mathrm{G}$ activity in wound fluid. Experimental dermatology 20 (6), 508-513. [PubMed: 21488974] 


\section{Highlights}

- Wound healing technologies constitute a major commercial enterprise, with the market for products involved in wound closure exceeding $\$ 15$ billion and the market for skin scar prevention accounting for another $\$ 12$ billion, but there are major differences among the wound care products used in clinical practice.

- Smart systems, which are devices with sensing, responding, or reporting functions, or a combination of these, can address many of the challenges associated with wound healing, particularly for chronic wounds.

- $\quad$ Smart systems may also promote better wound management, improving clinical outcomes by means such as detecting infections in a timely manner or providing alerts for patients. 
What other unmet needs exist in wound care?

How can smart systems be practically implemented to revolutionize wound care?

What different types of smart wound care products could be developed?

What are some unforeseen benefits of smart systems and challenges to be overcome? 


\section{Box 1.}

\section{Dysregulation of normal wound healing in chronic wounds}

In healthy individuals, skin heals in a predictable manner after injury through a cascade of interconnected molecular and cellular events [1,2]. Following a superficial wound, blood flow through site of injury is stopped through hemostasis. During the subsequent inflammatory phase, vascular permeability is increased, allowing enzymes, nutrients, and immune cells such as neutrophils, macrophages, and lymphocytes to reach the site of injury [4]. During the proliferative phase, fibroblasts move into the wound bed in response to growth factors and secrete a temporary extracellular matrix (ECM) serving as a scaffold for growth of other cells. The endothelial cells enter a rapid growth phase, while the granulation tissue experiences angiogenesis to supply this active metabolic area. [10]. During the final phase of remodeling or maturation, the vascular network is regressed and the ECM and collagen is replaced and remodeled extensively [4]. While in regular wounds the inflammatory phase of the wound healing process typically stops after days or a few weeks and is followed by a proliferation and tissue remodeling phase $[13,14]$, in some cases the inflammation phase can persist for months or even years, forming a chronic wound $[14,15]$. The wound candidates that are most likely to show slow healing are venous leg ulcers, pressure ulcers, and diabetic foot ulcers, with most of these wounds taking more than one year to heal [14]. 


\section{Box 2.}

\section{Limitations of current wound care technologies}

The majority of existing wound care dressings are passive and cannot actively respond to variations in the wound environment. In some cases, these passive dressings are able to release anti-inflammatory drugs, antibiotics or antibacterial compounds, angiogenic factors, and uptake excessive exudate [3]. Some of the more advanced dressings passively release biological factors and compounds to facilitate tissue healing [8]. One of the major limitations of current wound care products is their inability to provide information about the status of the wound bed and its healing rate. As a consequence, patients must be frequently screened to assess the healing process and inspect for potential infection. Increased visits, stemming from the necessity to continuously monitor the healing process, add to the cost of the treatment and enhance the stress on medical centers. In addition, frequent visits to medical centers can be a major challenge for patients who are living in remote areas. Another important limitation of passive wound care products is their inability to recognize the difference between various stages of wound healing. In general, the rates of physiological processes are different throughout the healing process and as a result the concentration of the required factors and drugs may vary over time. Other challenges include the correct utilization of antibiotics, where improper and prophylactic use can lead to the development of antibiotic-resistant bacteria. 


\section{Box 3.}

\section{Importance of some markers for assessing wound status}

pH

The $\mathrm{pH}$ of the wound bed is one of the significant biomarkers which can provide important information about the status of a wound throughout the healing process [5]. It has been reported that more than $80 \%$ of chronic wounds with elevated $\mathrm{pH}$ are infected. Skin is naturally acidic, with a $\mathrm{pH}$ value in the range of four to six. The acidic environment is thought to support proliferation of fibroblasts [6,7], promote angiogenesis and epithelialization [6,7], aid the release of oxygen from oxyhaemoglobin [11], and control bacterial colonization [16]. The $\mathrm{pH}$ of the tissue underlying skin is more neutral with a $\mathrm{pH}$ of 7.4. Thus, wounding will cause exposure of this underlying tissue and alter the acidic environment at the site of injury [7]. Also, chronic wounds often suffer from the cycles of ischemia-reperfusion injury and thus their $\mathrm{pH}$ value is higher than regular healing wounds. Such wounds are also usually colonized with environmental pathogens and once infected may become more basic to further optimize bacterial growth and have an elevated $\mathrm{pH}$ of up to 10 .

\section{Temperature}

Measuring temperature can provide information regarding local blood flow [19] and lymphocyte extravasation, along with wound infection and chronicity [21]. A randomized study found a correlation between increased temperature and increased angiogenesis and fibrosis [22]. A study of 35 patients with stage II-IV PUs found that ulcers with high temperatures healed more slowly than low temperature ulcers, where the higher temperatures may be indicating the presence of critical colonization [21]. Extreme hypothermia has also been correlated with unsuccessful wound healing and increased wound infection [24, 25]. Overall, temperature is a parameter that provides information on various factors relevant to healing, such as adequate blood flow, presence of infection, and oxygenation. As a result, temperature has been suggested as a marker that can be monitored in order to understand the level of inflammation or infection [21].

\section{Oxygen}

Oxygen is a requirement for the effective progression of the wound healing process, supporting the wound bed with cell proliferation, angiogenesis, collagen synthesis and bacterial defense [27]. Previous works have found conflicting results on the effects of oxygen in wound healing with some studies suggesting that hypoxia induces angiogenesis while others suggest that hypoxia enhances angiogenic cytokines [29]. Some studies suggested a minimum tissue oxygen tension of $20 \mathrm{mmHg}$ to promote wound healing, though this value was observed to be significantly lower in non-healing wounds [30]. Despite these varied statements, oxygen is important at different stages in wound healing and must be studied alongside other factors when assessing wounds.

\section{Moisture}

Inflammation resulting from the initial injury enhances capillary permeability and the leakage of fluid from blood vessels. The majority of the exudate is produced during the 
inflammatory and proliferative stages of wound healing, but its production rate varies depending on the stage of the healing and the characteristics of the wound [31, 32]. Wound healing in a moist environment also was found to have a greater rate of revascularization and dry wounds also had a slower progression to the remodeling phase of wound healing [34]. However, since some bacterial species grow better in a moist environment, excessive wound fluid may also increase the risk of bacterial infection [31]. Thus, measuring the level of moisture in the wound environment can provide information on the wound status and the proper intervention for managing the exudate and moisture level. 
(A)
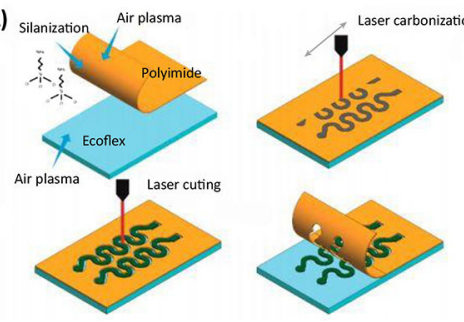

(D)

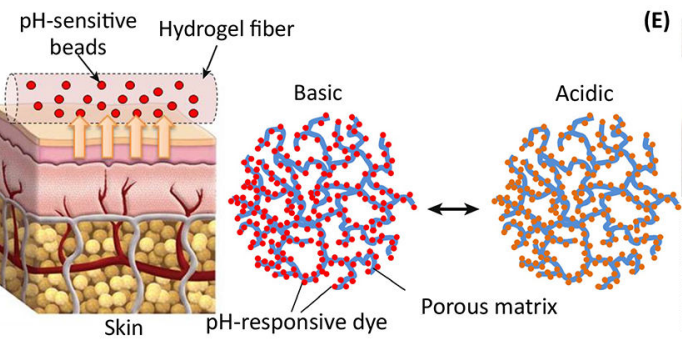

Spray coating

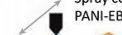
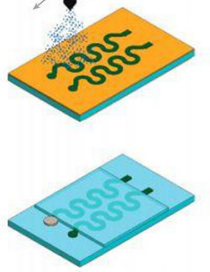

(B)

(E)

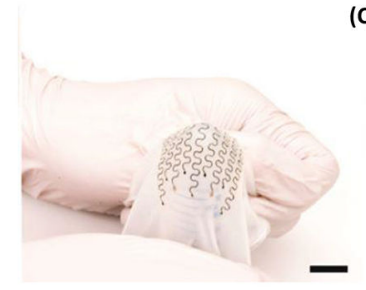

(c)
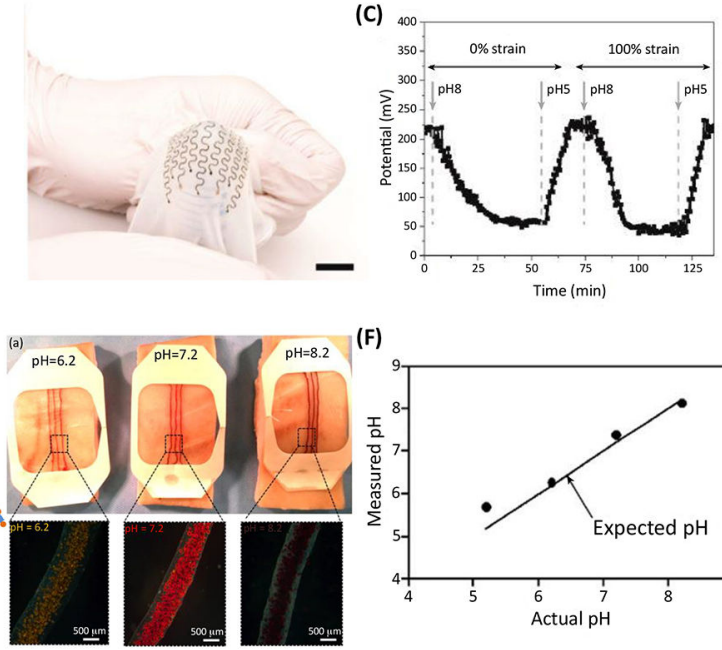

(F)

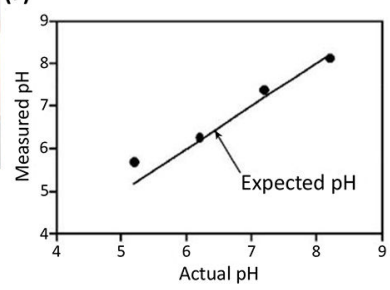

Figure 1.

Examples of bandages with integrated $\mathrm{pH}$ sensors. (a) Schematic view of the fabrication process of highly stretchable potentiometric $\mathrm{pH}$ sensors. (b) Photograph of a typical bandage with integrated $\mathrm{pH}$ sensor placed on a curved surface demonstrating its stretchability and flexibility. (c) Real-time monitoring of the $\mathrm{pH}$ and the sensor response to $\mathrm{pH}$ changes under various mechanical strains showing negligible effect of strain on the sensor readout. (d) A colorimetric $\mathrm{pH}$ sensor based hydrogels carrying beads loaded with $\mathrm{pH}$-sensitive dye for long-term monitoring of wound. (e) Images demonstrating color changes in of $\mathrm{pH}$ sensitive dressings on the pig skin when sprayed with solutions of different $\mathrm{pH}$ values. (f) The data extracted from the images taken using a smartphone in comparison to the actual $\mathrm{pH}$ values of the sprayed solutions. Figures are reproduced with permission from [39], [40]. 


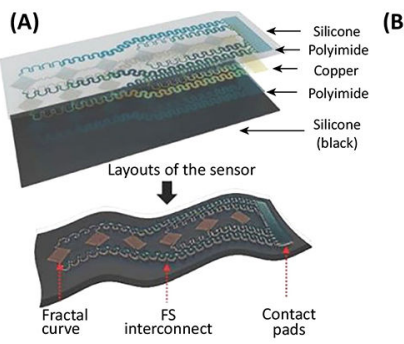

)

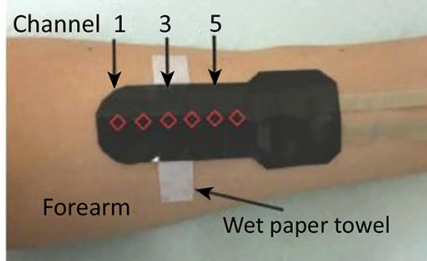

(E)

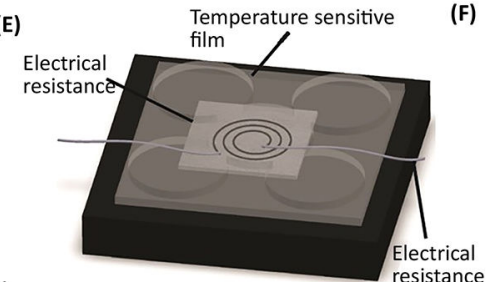

(H) (c)

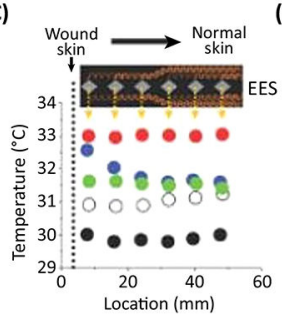

(G) 1.2

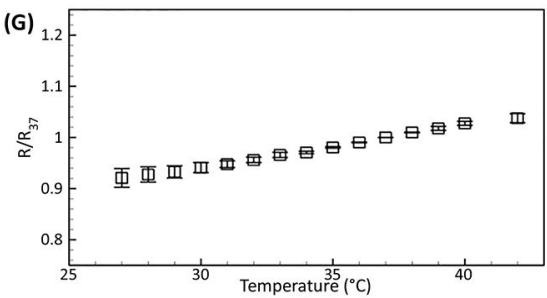

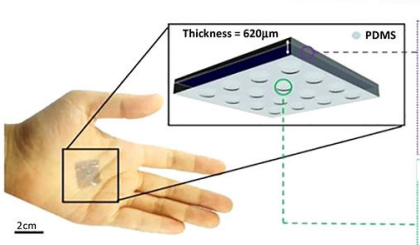

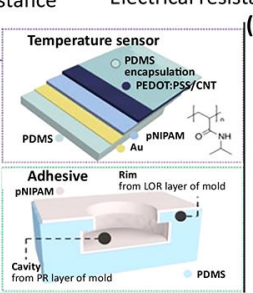

(K)

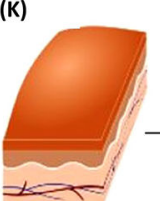

Apply as liquid

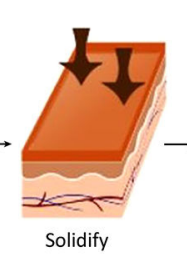

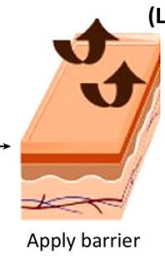

Apply barrier

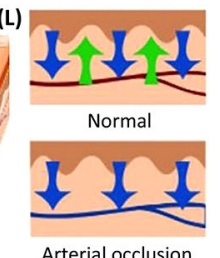

Arterial occlusion
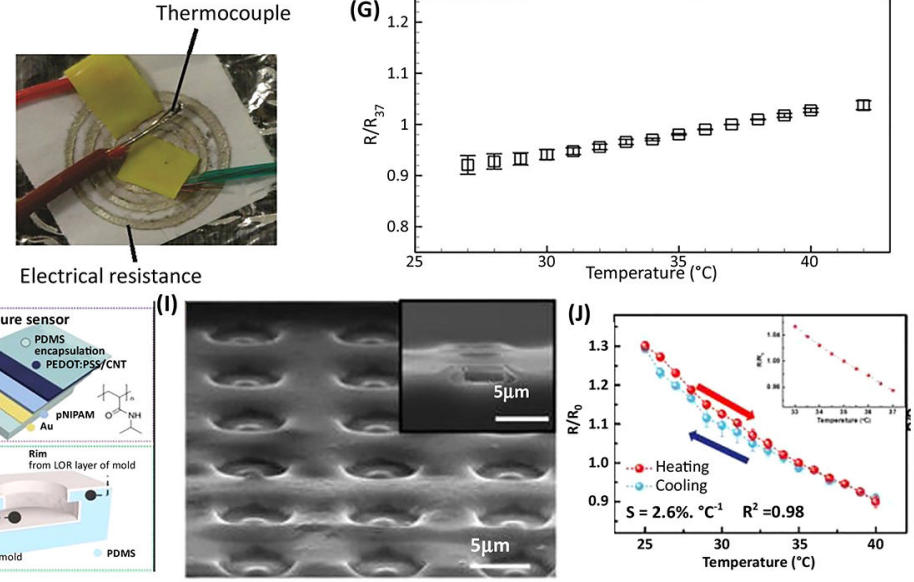

$\perp_{2}$ consumption $(M)$ $\mathrm{O}_{2}$ Replenishment
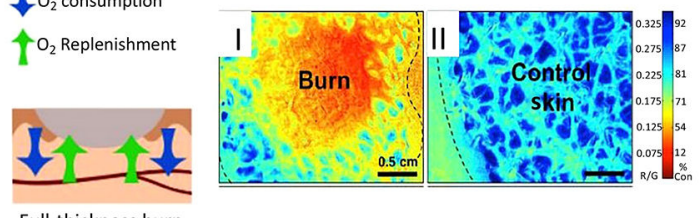

Figure 2.

Examples of wound dressings capable of monitoring skin temperature and oxygenation. (a)

Schematic illustration of microfabrication of flexible temperature sensor. (b) A typical fabricated bandage that could maintain conformal contact with the skin. (c,d) The distribution of cutaneous temperature and thermal conductivity measured using the bandage.

(e) Schematic illustration of a temperature sensor fabricated on an elastic nanofibrous substrate. (f) A representative image of a sensor fabricated by screen printing of silver ink on the nanofibrous substrate. ( $\mathrm{g}$ ) Changes in the relative electrical resistance of the fabricated sensor normalized to its reference resistance at $37^{\circ} \mathrm{C}$. (h) Image of the wearable temperature sensor attached on the skin and schematic illustration of the different layers of the fabricated temperature sensors. (i) A SEM image of the octopus mimicking surface applying suction for enhanced adhesion to the skin surface and forming conformal contact. (j) The variation of the relative resistance of the integrated sensor with temperature. (k) Schematic illustration of the application of the oxygen sensing of liquid-based bandage. (1) Schematic demonstrating of the tissue oxygenation in various types of wounds. (m) Oxygen consumption measured using the bandage in burn tissue (I) and healthy tissue (II). Blue depicts higher oxygen consumption and red indicates lower oxygen consumption in the tissue. Figures are reproduced with permission from [44], [42], [57], [45]. 

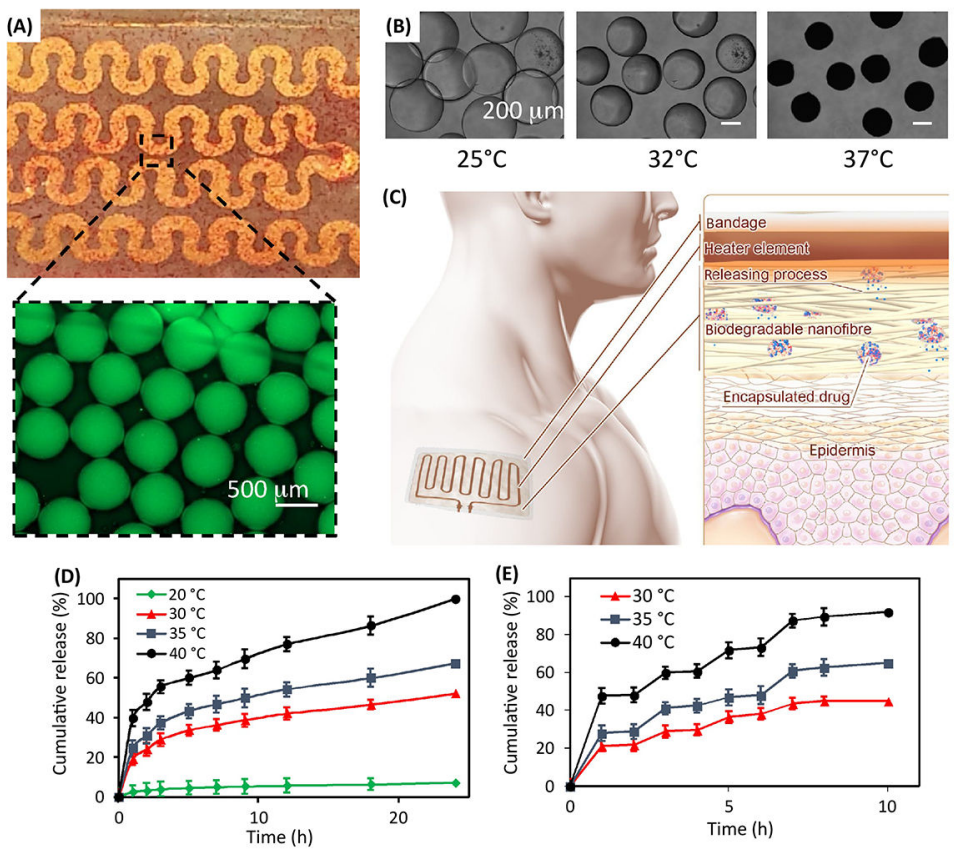

(F) Conductive fiber
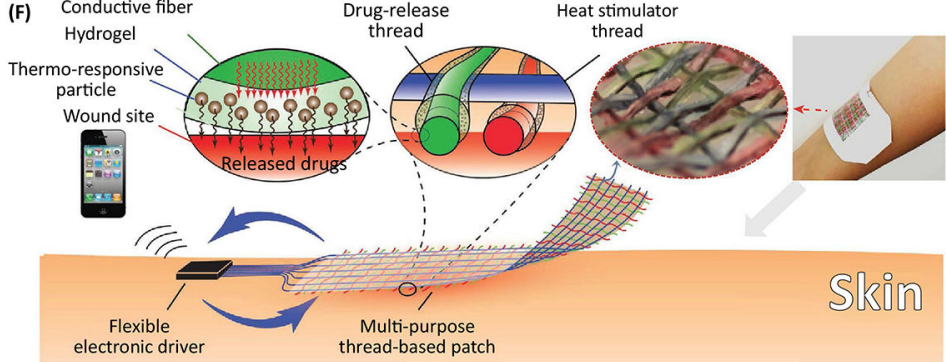

(G)
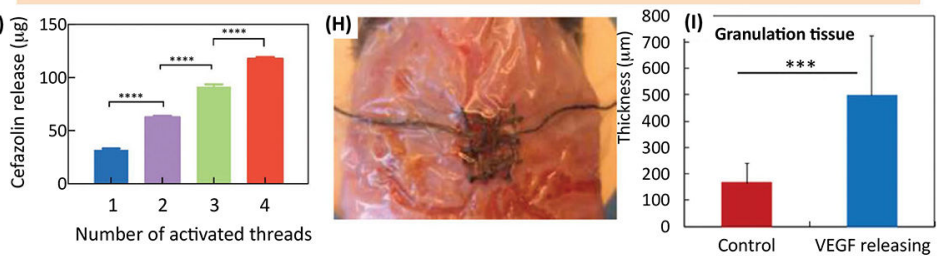

Figure 3.

Examples of bandages enabling active control over drug release. (a) Photograph and micrograph of thermoresponsive drug carriers encapsulated in an alginate layer casted on a flexible heater. (b) The response of the engineered thermo-responsive particles to the increase of temperature leading the release of encapsulated compounds. (c) The schematic showing the operation principle of the engineered platform with the integrated heater and electronics. Thermo-responsive drug nanocarriers were encapsulated in nanofibrous mesh fabricated through electrospinning. These particles released their payload in response to temperature increase induced by the integrated flexible heater. (d) The release profile of cefazolin from the nanofibrous mesh at four different temperatures. (e) Effect of temperature on the release rate of ceftriaxone encapsulated within the nanofibrous mesh upon cyclic heating. (f) Schematics of a thread-based patch enabling active control over the release of different drugs. Each fiber consisted of a core heater, coated by a hydrogel layer carrying 
thermo-responsive particles. $(\mathrm{g})$ The effect of number of activated fibers on cefazolin release from a textile patch. (i) Image of the patch on the wound model. (h,i) A typical fabricated bandage used for releasing VEGF into wounds in diabetic mice. The VEGF delivery improved wound healing rate and tissue granulation. Figures are reproduced with permission from [67], [68], [69]. 
(A)

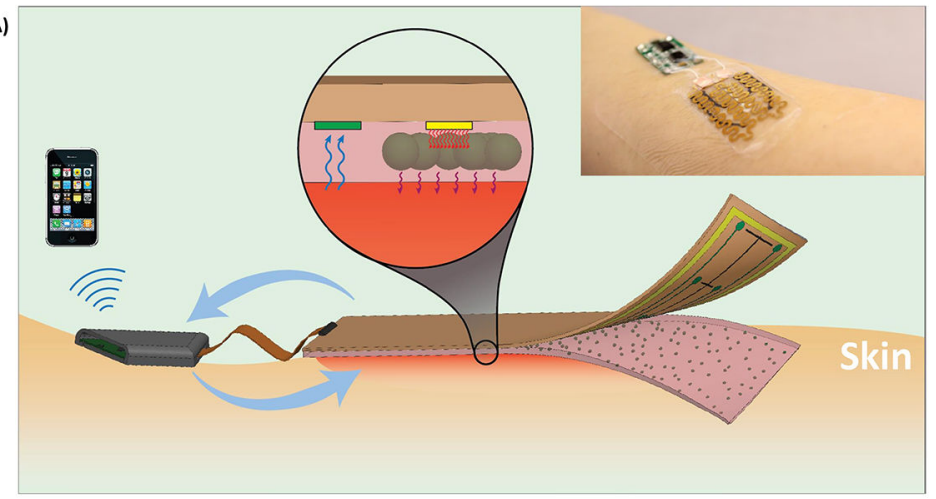

(B)

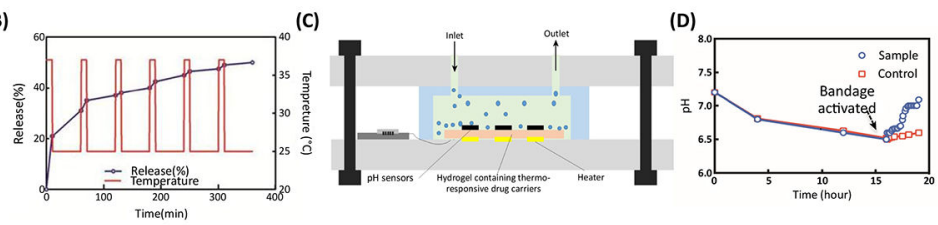

(E)

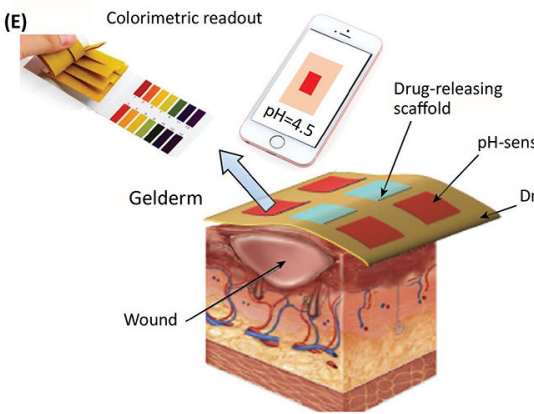

(F)

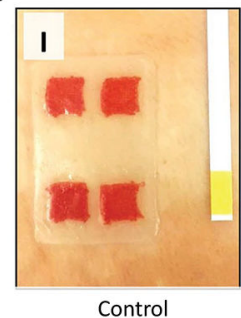

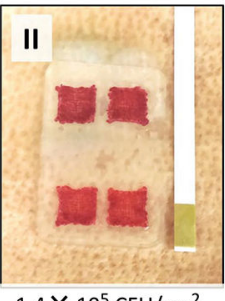

$1.4 \times 10^{5} \mathrm{CFU} / \mathrm{cm}^{2}$

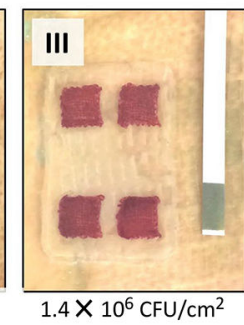

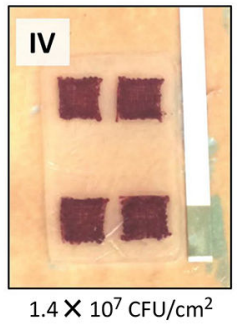

Figure 4.

Smart and automated bandages for treatment of chronic wounds. (a) Schematic and a representative image of multi-layer dressing, which was capable of sensing the wound $\mathrm{pH}$ and releasing antibiotics in response to the activation of an integrated heater. The sensing data was processed on board and the heater could be activated in response to the changes in the environmental condition. (b) Cyclic activation of the heater and the variation in drug release in response. (c) Schematics of an in vitro model utilized for culturing of Staphylococus aurous in a bioreactor interfaced with the bandage. (d) The bandage was continuously monitoring the bioreactor $\mathrm{pH}$ and once a critical $\mathrm{pH}$ was detected, antibiotics were released. The eradication of bacteria followed by continuous perfusion led to recovery of the bioreactor $\mathrm{pH}$. (e) Schematic of a semi-automated bandage with colorimetric $\mathrm{pH}$ sensor and drug delivery capability. (f) The bacterial growth could affect the color of the 
engineered bandage, as shown by the darkening color in (I)-(IV) as CFU increases. Figures are reproduced with permission from [74], [75].1 
Table 1.

List of markers with applications in engineering smart wound dressings

\begin{tabular}{|l|l|l|}
\hline (Bio)marker & Significance & References \\
\hline pH of wound & Understanding wound status. For example, elevated pH in wound bed is a sign of infection. & [39-41] \\
\hline Temperature & $\begin{array}{l}\text { Providing information on various factors relevant to healing, infection, inflammation and } \\
\text { oxygenation in the wound environment. }\end{array}$ & [42-44] \\
\hline Oxygen & $\begin{array}{l}\text { Hypoxia in a chronic wound may be detrimental to the healing process. The oxygen level also } \\
\text { indicates the rate of angiogenesis. }\end{array}$ & [45, 46] \\
\hline Moisture & $\begin{array}{l}\text { Moisture in the wound bed reduces the inflammation and enhances the proliferative phase of } \\
\text { wound fluid may increase the risk of bacterial infection. }\end{array}$ & [47] \\
\hline Mechanical and electrical & $\begin{array}{l}\text { The mechanical and electrical properties of skin or wound change in response to skin disorders or } \\
\text { diseases as well as during the healing process. }\end{array}$ & [42, 48] \\
\hline Enzymes & $\begin{array}{l}\text { The upregulation or downregulation of enzyme levels can indicate abnormalities. For example, the } \\
\text { upregulation of cathepsin G and elastase has been suggested as indicators of infection. }\end{array}$ & [49] \\
\hline
\end{tabular}

